\title{
Sensitivity Analysis for Linear Systems based on Reachability Sets
}

\author{
Daniel Silvestre, Paulo Rosa, João P. Hespanha, Carlos Silvestre
}

\begin{abstract}
The problem of deciding which inputs in a model influence the most the state or output is often of practical importance, especially in the cases in which the system can be over-parameterized. In this context, a designer is required to perform sensitivity analyses so as to select which inputs are the most relevant to the problem at hand and remove those with smaller or no impact. In this paper, we tackle this issue by constructing the exact reachable set of a linear system that relates the inputs with the state of that system. By means of projections and solutions of linear optimization programs, we are able to assess which inputs drive the most the state or the output of a linear system. Illustrative examples are presented in order to provide insights on the proposed method.
\end{abstract}

\section{INTRODUCTION}

Sensitivity analysis has been a long standing research topic addressed by multiple techniques. The problem relates to the identification of the inputs causing the largest variability of the state/output of a model. Different techniques have been proposed and lengthy discussions are presented in the books [1], [2], [3], while further information can be found in the review articles [4], [5].

The sensitivity analysis is typically conducted by defining a model, assigning probability density functions to each input, generating inputs, and assessing the output of the model. The view in this paper is centered on a worstcase scenario where, instead of considering the probability information, the sensitivity analysis is driven by the most extreme impact each input can have on the state/output of the model.

The main motivation of considering the sensitivity of a model is to find which inputs are the most successful in achieving a control strategy or which contribute the most to the outputs of the system. We envisage as particular case of interest a formation of agents with their own dynamics.

D. Silvestre is with the Department of Electrical and Computer Engineering of the Faculty of Science and Technology of the University of Macau, Macau, China, and with the Institute for Systems and Robotics (ISR), Instituto Superior Técnico, University of Lisbon, Lisbon, Portugal. D. Silvestre was supported by the project MYRG2016- 00097-FST from the University of Macau, by the Portuguese Fundação para a Ciência e a Tecnologia (FCT) through Institute for Systems and Robotics (ISR), under Laboratory for Robotics and Engineering Systems (LARSyS) project UID/EEA/50009/2019. dsilvestreeisr.tecnico.ulisboa.pt

P. Rosa is with Deimos Engenharia, Lisbon, Portugal. paulo.rosa@deimos.com.pt.

C. Silvestre is with the Department of Electrical and Computer Engineering of the Faculty of Science and Technology of the University of Macau, Macau, China, on leave from Instituto Superior Técnico/Technical University of Lisbon, 1049-001 Lisbon, Portugal. The work was supported by project MYRG2016- 00097-FST of the University of Macau. csilvestre@umac.mo

João P. Hespanha is with the Dept. of Electrical and Computer Eng., University of California, Santa Barbara, CA 93106-9560, USA. This research was partially funded by the NSF grants no EPCN-1608880 and CNS1329650. hespanha@ece.ucsb.edu
Which nodes contribute the most for a final decision depends on their individual dynamics but also on the topology. The techniques designed for sensitivity analysis in this paper aim to answer the question of which inputs should be used or, in the opposite direction, which elements are the ones that can cause the final state to drift the most in the worst-case scenario. In a smart grid environment, such techniques would be useful to determine what is the worst operating point if one of the inputs is compromised or faulty following the concepts in [6].

The first group of methods that has been used to address the problem can be categorized as direct methods or differential sensitivity analysis because the model equations are differentiated (similarly computed the difference equations) with respect to the inputs. The impact of each input can then be described by these derivatives. Many formulations exist for continuous-time models that are surveyed in [5] and that have been more recently developed in the works [7], [8], and also for the discrete-time case in [9], by exploiting the case of a Kalman filter.

The variance-based methods in [7], [8] determine the sensitivity of each input by computing the variance on the output caused by the inputs through an approximation model, by using Taylor series expansions. These methods have the advantage of considering a general nonlinear model of the type $Y=f\left(X_{1}, \cdots, X_{k}\right)$, whereas the focus of this paper is on the linear case and with the main difference that only the variance is being used to compute the sensitivity. Our proposal is to leverage on recent developments in reachability sets computational approaches as a measurement of the uncertainty or variability caused by a given input.

Additional methods exist that estimate the variance using for example FAST (Fourier Amplitude Sensitivity Test) [10], which resorts to the Fourier series to represent the ANOVA decomposition of the nonlinear model. Similarly, some also use the WASP (Walsh Amplitude Sensitivity Procedure) [11]. The main objective is to compute the ratio between the variance of $Y$ given $X$ for all possible values of $X$ and the variance of $Y$ as a measure of the sensitivity. Examples range from [12], in which a transformation is used to reduce the computational cost, to [13] for a general sensitivity analysis independent of the model and also [14] where different methods based on variance using FAST are compared with direct methods. All such techniques consider the sensitivity from a variance point-of-view, trying to identify which inputs cause the most variability. Another interesting question arises when focusing on the support of the distribution where finding which input generates the worst possible scenario among all the plant inputs.

In [15], the authors show how the sensitivity can also be 
computed from the posterior probability given a prior on the inputs and thus describing it by means of a Bayesian approach. Using the previous method requires fewer runs than data-driven techniques such as Monte-Carlo tools. The work in [16] addresses the case of determining the sensitivity of medical parameters in a model by a Monte-Carlo approach, where the input space is sampled and propagated with the model, so as to determine its variance. The case of the over-parameterized hydrological models is also studied in [17] using a latin-hypercube sampling and a one-factor-at-atime (OAT) sampling to produce a global sensitivity analysis. Other applications examples are: water flow and quality in [18]; the spread of malaria in [19]; assessing technology according to the National Institute for Clinical Excellence in [20]; hybrid systems in [21]; and the interested reader is referred to the recent survey in [22] for further information.

In this paper, the focus is on constructing reachability sets for linear systems in order to quantify the impact of a given input on the state/output of the model. Intuitively, the variability of the state/output with respect to the inputs is measured by computing their respective interval through a reachable set. Resorting to the concept of Set-Valued Observers (SVOs) enables the generation of a polytope representing the restrictions on the state given the inputs and uncertainties on the initial state. The choice satisfies the need for an optimal representation for linear systems (i.e., no conservatism is added when there are no uncertainties in the dynamics). Motivated by the findings in [23] that OAT strategies might be justified for linear models, we sought to investigate that claim via a direct comparison between the two approaches, i.e., considering a single input versus considering multiple inputs simultaneously. The contributions of this paper can therefore be summarized as follows:

- The introduction of a formal method that makes use of reachability sets to compute the sensitivity of each input;

- A result proving, for linear models, the relationship between the sensitivity of OAT input with testing all the inputs at the same time.

Notation : The transpose of a matrix $A$ is denoted by $A^{\top}$. For vectors $a_{i},\left(a_{1}, \ldots, a_{n}\right):=\left[\begin{array}{lll}a_{1}^{\top} & \ldots & a_{n}^{\top}\end{array}\right]^{\top}$. We let $1_{n}:=[1 \ldots 1]^{\top}$ and $0_{n}:=[0 \ldots 0]^{\top}$ indicate $n$-dimensional vector of ones and zeros, respectively, and $I_{n}$ denotes the identity matrix of dimension $n$. Dimensions are omitted when clear from context. The vector $e_{i}$ denotes the canonical vector whose components are equal to zero, except for the $i$ th component. The symbol $\otimes$ denotes the kronecker product.

\section{REACHABILITY FOR LINEAR SYSTEMS}

In the context of sensitivity analysis, one is typically interested in determining what happens to the output if the inputs belong to some hypercube. Assuming a linear system, the optimal approach to model the smallest reachable set is using polytopes, as demonstrated in [24]. Therefore, we consider a definition that follows the same principles as those of Set-valued Observers (SVOs) that can be found in recent works [25], [26], [27], [28] and the references therein.
We consider a linear time-invariant model of the form:

$$
\begin{aligned}
& x(k+1)=A x(k)+B u(k)+E d(k), \\
& y(k)=C x(k)
\end{aligned}
$$

where $x(k) \in \mathbb{R}^{n_{x}}, y(k) \in \mathbb{R}^{n_{y}}, u(k) \in \mathbb{R}^{n_{u}}$ and $d(k) \in$ $\mathbb{R}^{n_{d}}$, with matrices of appropriate size. In order to build a closed reachability set, we assume the bound $\forall_{1 \leq i<n_{d}}$ : $\left|d_{i}(k)\right| \leq 1$ without loss of generality since matrix $E$ can be appropriately scaled. The main objective is to characterize the impact of each entry of input $d(\cdot)$ to the state $x(k)$ or the output $y(k)$.

To review the steps in the construction of reachable sets from the model in (1), we $\operatorname{define} \operatorname{Set}(M, m):=\{q: M q \leq$ $m\}$, which represents a convex polytope, with the operator $\leq$ being a component-wise operation between the two vectors. The aim of an SVO (Set-Valued Observer) is to find the smallest set $X(k)$ containing all possible states of the system at time $k$, knowing that $\forall_{0<i<H}, x(k-i) \in X(k-i)$ for all past $H$ time steps and the dynamics of the system (1) for all possible values of inputs $d(k)$.

More precisely, the initial state satisfies $x(0) \in X(0)$, where $X(0):=\operatorname{Set}\left(M_{0}, m_{0}\right)$ and $M_{0}$ and $m_{0}$ are selected such that the corresponding polytope is guaranteed to contain the initial state. The notation $\bar{Z}:=\left[\begin{array}{c}Z \\ -Z\end{array}\right]$, for a matrix $Z$, and $\bar{v}:=\left[\begin{array}{c}v \\ -v\end{array}\right]$, for a vector $v$ will be used to shorten the following equations. The information obtained by an additional output measurement $y(k+1)$, results in a set $X(k+1)$ that can be described as the set of points, $\mathbf{x}$, satisfying

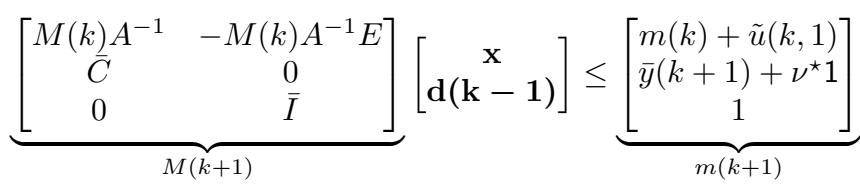

for some $\mathbf{d}(\mathbf{k}-\mathbf{1})$ where we used the notation $\tilde{u}(k, H):=$ $\sum_{\tau=1}^{H} M(k) A^{-\tau} B u(k-\tau+1)$.

We will be assuming an invertible matrix of the dynamics $A$ to enable the previous strategy, although it is possible to construct the same set otherwise by resorting to the strategy in [24].

The above computations assume a horizon value $H=1$, i.e., only the measurements from time $k+1$ and the input signal from time $k$ are used to compute the set-valued estimate of the state at time $k+1$. One can leverage on this idea to construct the set corresponding to the constraints that respect the model from time zero to some time $k$, since this will be suitable for the purpose of sensitivity analysis. The computations can be extended to a general horizon $H$ by defining the set as:

$$
M(H)\left[\begin{array}{c}
\mathbf{x} \\
\mathbf{d}(\mathbf{0}) \\
\vdots \\
\mathbf{d}(\mathbf{H}-\mathbf{1})
\end{array}\right] \leq m(H)
$$


where

$M(H):=\left[\begin{array}{c|ccc}M_{0} A^{-H} & -M_{0} A^{-1} E & \ldots & -M_{0} A^{-H} E \\ 0_{2 H n_{d} \times n_{x}} & & I_{H} \otimes \bar{I}\end{array}\right]$, $m(H):=\left[\begin{array}{c}m_{0}+\hat{u}(H) \\ 1_{2 H n_{d}}\end{array}\right]$

with $\hat{u}(H):=\sum_{\tau=1}^{H} M_{0} A^{-\tau} B u(\tau-1)$.

The inequality in (2) describes an optimal polytope that contains all constraints given by the model (1) and the hypercube for inputs $d(0), d(1), \cdots d(H)$ for which we would like to analyze the associated contributions to the state. The relationship between the state $x(H)$ and the values of $d(\cdot)$ are captured in the polytope.

\section{Sensitivity Analysis using Polytopes}

The general sensitivity analysis considers the individual contributions as well as the possible interactions among the inputs as opposed to the OAT framework where each input is taken independently. In this section, the two cases will be addressed.

\section{A. General Sensitivity}

The set $X(H)$ defined by (2) comprises all points of the type $(x(H), d(0), \cdots, d(H-1))$ that satisfy the dynamics (1) and can be reached for at least a point on the set of values being tested for the inputs. This property holds even if the reachable set $X(H)$ is not convex. Using the general sensitivity approach, one can project the set into a the lower dimension set defining how $x(H)$ varies with respect to a subset of the inputs, while setting the remaining to zero. The sensitivity of the state or output corresponds to the interval between the maximum and the minimum value attained with a worst-case selection. This formulation admits more general linear models and the addition of constraints to the set of admissible states. For instance, one can answer questions like "what is the sensitivity of state $j$ at time $H$ with respect to inputs 2 and 3 , given some unknown initial condition, and in the case where the measurement at time $H-5$ is zero?".

Returning to the assumptions in this paper, since $X(H)$ is a polytope, one could resort to the so-called Fourier-Motzkin elimination method [29] to remove the dependence on some of the inputs and obtain the description of $x(H)$ on a smaller subset. However, such tools have a heavy computational complexity and, since the objective is solely to compute the amplitude of the state or output with respect to the admissible values of the inputs, a different technique is proposed in this paper.

Since the computation of the sensitivity is going to be written as an optimization problem, we will constrain the state to belong to the space with a single non-zero $i$ th input. In order to do so, we introduce the linear map $\Pi_{i}: \mathbb{R}^{n_{x}+1} \mapsto$ $\mathbb{R}^{n_{x}+H n_{d}}$ defined as

$$
\Pi_{i}(x)=\left[\begin{array}{cc}
I_{n_{x}} & 0 \\
0 & \mathrm{e}_{i}
\end{array}\right] x
$$

where $\mathrm{e}_{i}$ is the $i$ th vector of the canonical basis of $\mathbb{R}^{H n_{d}}$, and is helpful in formulating the problem of finding the sensitivity amplitude for input $i$, where $1 \leq i \leq H n_{d}$. However, it can be generalized to select more than one input by replacing $e_{i}$ with a matrix $\left[\begin{array}{lll}e_{i_{1}} & e_{i_{2}} & \cdots\end{array}\right]$ to select inputs $i_{1}, i_{2}, \cdots$. Thus, the selection can take inputs at different time instants or entries in the input vector.

Let the notation $X_{i}(H):=\left\{x: \Pi_{i}(x) \in X(H)\right\}$ represent the projected set, which will not be computed explicitly. Remark that input $i$ is an entry of the input vector at a given time and therefore there are $H n_{d}$ inputs. Then, the sensitivity function can be given as in the following definition.

Definition 1: Given a set $X(H)$ built for a given horizon $H$, the general sensitivity of state $j$ to the input $i$ can be defined as the function:

$$
\begin{aligned}
& \mathcal{S}\left(X_{i}(H), j\right):=x_{j}^{\max }(H)-x_{j}^{\min }(H), \\
& x_{j}^{\max }(H)=\max _{j} x_{j}(H) \\
& x_{j}^{\min }(H)\left.=\min _{d_{i}}^{x(H)}\right]_{\in X_{i}(H)} x_{j}(H) . \\
& {\left[\begin{array}{c}
x(H) \\
d_{i}
\end{array}\right] \in X_{i}(H) }
\end{aligned}
$$

In Definition $1, d_{i}$ is an input where $1 \leq i \leq H n_{d}$. Notice that this function measures the sensitivity of the state (similarly the definition can represent an output if the polytope is constructed for the output) to the $i$ th input. The problem can be solved independently by computing two linear optimization programs:

$$
\begin{array}{cl}
\underset{z}{\operatorname{minimize}} & {\left[\begin{array}{c}
\mathrm{e}_{j} \\
0_{n_{d}}
\end{array}\right]^{\top} z} \\
\text { subject to } & z=\Pi_{i}(x), \\
& M(H) z \leq m(H) .
\end{array}
$$

and

$$
\begin{array}{cl}
\underset{z}{\operatorname{minimize}} & -\left[\begin{array}{c}
\mathrm{e}_{j} \\
0_{n_{d}}
\end{array}\right]^{\top} z \\
\text { subject to } & z=\Pi_{i}(x), \\
& M(H) z \leq m(H) .
\end{array}
$$

The optimization programs (3) (minimum) and (4) (maximum) directly compute the two terms in function $\mathcal{S}_{i}(X(H), j)$. Remark that the solution of both problems is a linear objective function in a projected space from the original reachability set, which can be extended to other ways of computing the sets and more general models than that in (1). The steps are summarized in Algorithm 1.

The next lemma proves the intuition stated in [23], clarifying the relationship to the dynamics applied to the initial state.

Lemma 1 (General sensitivity for Linear Systems): Consider an initial state $x_{0}$ satisfying $\left\|x_{0}\right\|_{\infty} \leq 1$ for the linear system in (1) and a vector of inputs $d$. Also consider the definition of $\mathcal{S}\left(X_{i}(H, \mathcal{J}), j\right)$ as the sensitivity amplitude of the state $x_{j}(H)$ to input $i$ assuming all inputs in $\mathcal{J}$ are zero. 


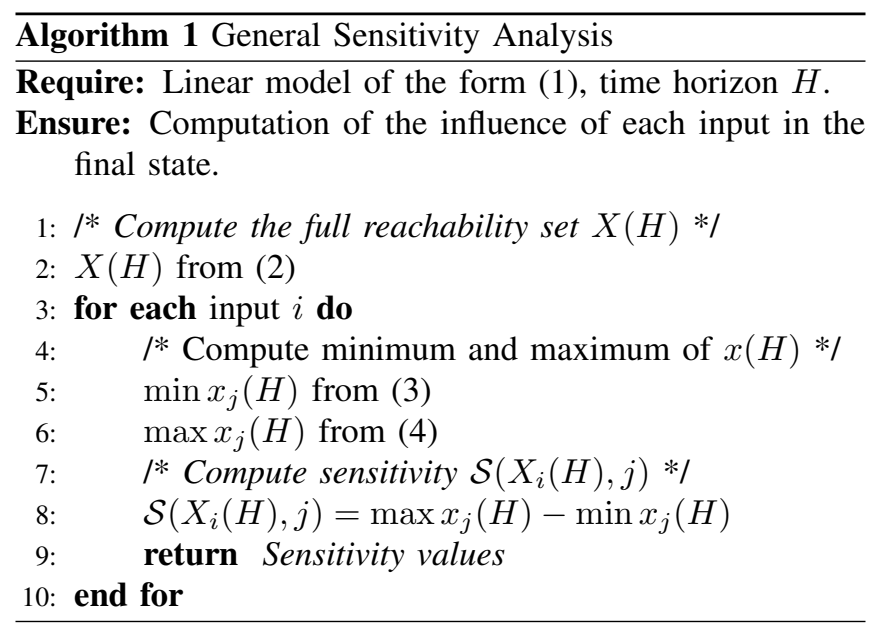

Then, the following holds:

$$
\mathcal{S}(X(H, \emptyset), j)=\sum_{\ell \in \mathcal{D}} \mathcal{S}\left(X_{\ell}(H, \mathcal{D} \backslash\{\ell\}), j\right)-(|\mathcal{D}|-1) \mathcal{S}_{x_{0}} .
$$

where $\mathcal{D}=\left\{\kappa \leq H n_{d}\right\}$ with integer $\kappa$, and $\mathcal{S}_{x_{0}}$ is the worstcase contribution of the initial state on state $x_{j}$ at time $H$, i.e.:

$$
\mathcal{S}_{x_{0}}:=\mathcal{S}(X(H, \mathcal{D}), j) .
$$

Proof: Let us write the solution to the state equation in (1):

$$
x(H)=A^{H} x_{0}+\sum_{\tau=0}^{H-1} A^{H-1-\tau}(E d(\tau)+B u(\tau)) .
$$

To compute $\mathcal{S}(X(H, \emptyset), j)$, given the bounds for the inputs and the initial state $\left\|x_{0}\right\|_{\infty} \leq 1$, one needs to compute

$$
\begin{array}{ll}
\underset{z}{\operatorname{argmin}} & \mathrm{e}_{j}^{\top} \mathcal{L}(z) \\
\text { subject to } & \|z\|_{\infty} \leq 1 .
\end{array}
$$

and

$$
\begin{array}{ll}
\underset{z}{\operatorname{argmax}} & \mathrm{e}_{j}^{\top} \mathcal{L}(z) \\
\text { subject to } & \|z\|_{\infty} \leq 1 .
\end{array}
$$

using different linear functions $\mathcal{L}(z)$. In particular, when $\mathcal{L}(z)=A^{H} z$ the optimization programs in (6) and (7) give us $x_{\min }$ and $x_{\max }$ as the arguments that minimize and maximize that linear function subject to the constraints. Therefore,

$$
\mathcal{S}_{x_{0}}=\mathrm{e}_{j}^{\top} A^{H}\left(x_{\max }-x_{\min }\right)
$$

as the amplitude of the sensitivity caused by the dynamics on the (unknown) initial state. Given that the objective function in the maximization of the $j$ th entry of the state vector in (5) is a separable function (i.e., linear), it holds:

$$
\mathcal{S}(X(H, \emptyset), j)=\mathcal{S}_{x_{0}}+\mathrm{e}_{j}^{\top}\left(\sum_{\tau=0}^{H-1} A^{H-1-\tau} E\left(d_{\max }^{\tau}-d_{\min }^{\tau}\right)\right)
$$

where $d_{\max }^{\tau}$ and $d_{\min }^{\tau}$ are the respective solutions to (7) and (6) with $\mathcal{L}(z)=A^{H-1-\tau} E z$. If we redo the calculations for and

$\mathcal{S}\left(X_{\ell}(H, \mathcal{D} \backslash\{\ell\}), j\right)$, and since all inputs are set to zero except $\ell$, one gets that:

$$
\left.\mathcal{S}\left(X_{\ell}(H, \mathcal{D} \backslash\{\ell\}), j\right)=\mathcal{S}_{x_{0}}+\mathrm{e}_{j}^{\top} A^{H-1-\tau} E\left(d_{\max }^{\ell}-d_{\min }^{\ell}\right)\right)
$$

i.e., it is the same state solution as in (5) but with all inputs except $\ell$ set to zero. Thus, the conclusion follows by noticing two facts. The first one is that $\mathcal{S}(X(H, \emptyset), j)$ is the sum of $\mathcal{S}_{x_{0}}$ and one term for each input $\ell$. Second fact is that summing all $\mathcal{S}\left(X_{\ell}(H, \mathcal{D} \backslash\{\ell\}), j\right)$ equals to adding $|\mathcal{D}|$ times the term $\mathcal{S}_{x_{0}}$ and the same terms related with the inputs $\ell$

The previous result asserted a relationship between the general sensitivity and the OAT strategy where the key step was a result of the linearity of (1). The next corollary reaches the same intuitive conclusion provided in [23].

Corollary 1: Assume that the initial condition $x_{0}$ is known, then the general sensitivity is the sum of the OAT sensitivities of each input.

Proof: The result follows from the fact that if $x_{0}$ is known then $x_{\max }=x_{\min }$ and $\mathcal{S}_{x_{0}}=0$.

Given the relationship between the general sensitivity and the OAT strategy for linear systems, in the next section further details are provided on how to compute this sensitivity for linear systems in an efficient manner.

\section{B. One-Factor-At-A-Time (OAT)}

The discussion about the general sensitivity pointed towards the adoption of an OAT strategy to the linear case. As a consequence, the computational complexity of the method is largely reduced because the number of variables is much smaller. Intuitively, OAT aims at fixing all inputs except one and computing the amplitude of change on the state caused by the analyzed input.

For simplicity of notation, let us say that the parameter $i$ corresponds to the entry $\ell$ at time $t$, i.e., that the labeling $d_{i}=$ $d_{\ell}(t)$. Fixing all but the $i$ th input to zero enables rewriting the polytope definition in (2) as:

$$
M_{i}(H)\left[\begin{array}{c}
\mathbf{x} \\
\mathbf{d}_{\mathbf{i}}
\end{array}\right] \leq m_{i}(H)
$$

where

$$
\begin{aligned}
& M_{i}(H):=\left[\begin{array}{c|c}
M_{0} A^{-H} & -M_{0} A^{t-1} E \mathrm{e}_{\ell} \\
0_{1 \times n_{x}} & 1 \\
0_{1 \times n_{x}} & -1
\end{array}\right], \\
& m_{i}(H):=\left[\begin{array}{c}
m(k)+\hat{u}(H) \\
1_{2}
\end{array}\right] .
\end{aligned}
$$

In turn, the optimizations required for computing the sensitivity also simplify to:

$$
\begin{array}{ll}
\underset{x}{\operatorname{minimize}} & {\left[\begin{array}{c}
\mathrm{e}_{j} \\
0_{n_{d}}
\end{array}\right]^{\top} x} \\
\text { subject to } & M_{i}(H) x \leq m_{i}(H) .
\end{array}
$$

$\underset{x}{\operatorname{minimize}}-\left[\begin{array}{c}\mathrm{e}_{j} \\ 0_{n_{d}}\end{array}\right]^{\top} x$

subject to $M_{i}(H) x \leq m_{i}(H)$. 


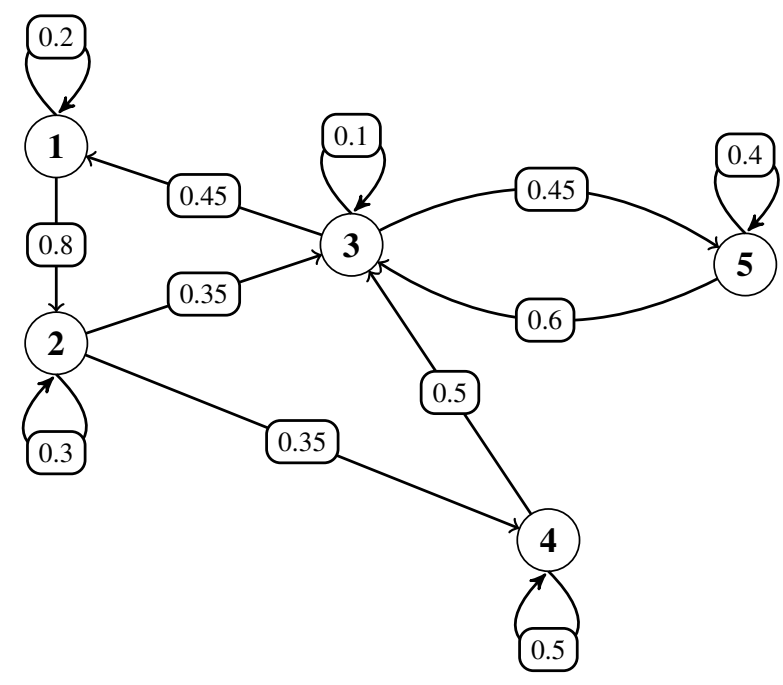

Fig. 1. Communication graph between the different vehicles.

Notice that both (9) and (10) do not involve any projection and work directly on the much smaller space of $\mathbb{R}^{n_{x}+1}$ instead of $\mathbb{R}^{n_{x}+H n_{d}}$. For comparison with Algorithm 1, it is summarized the steps of the OAT approach in Algorithm 2.

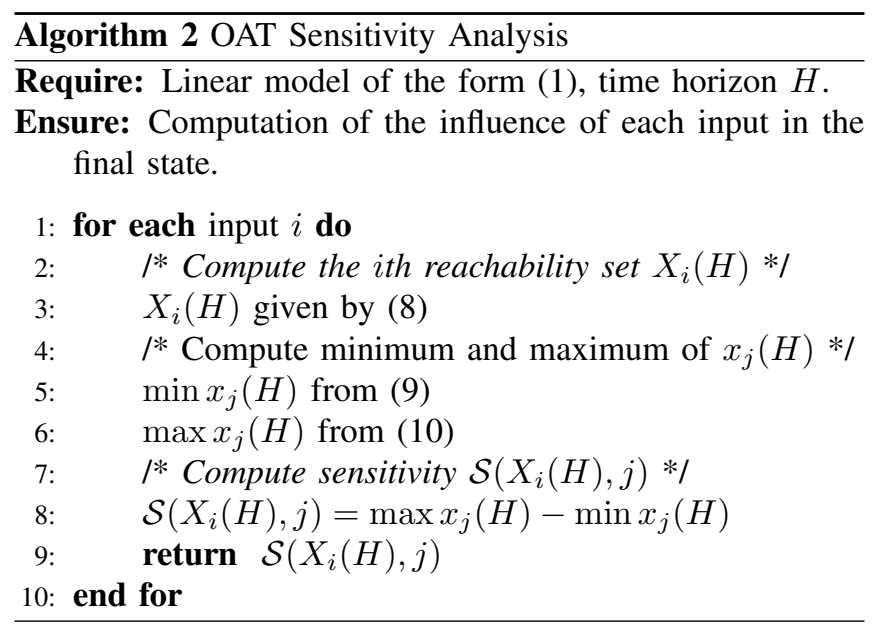

\section{Simulation Results}

In this section, we present simulations for a network comprised of $N=5$ vehicles with unicycle dynamics described in [30]. The formation follows the graph in Figure 1 where it is annotated the weights that each vehicle uses in a directional consensus algorithm to decide on a group velocity and direction values.

As described in [30], the discrete-time model for the $i$ th vehicle can be written as:

$$
\left[\begin{array}{c}
p_{i} \\
q_{i}
\end{array}\right](k+1)=\left[\begin{array}{c}
p_{i} \\
q_{i}
\end{array}\right](k)+T A_{i}\left(\theta_{i}\right)\left[\begin{array}{c}
v_{i} \\
w_{i}
\end{array}\right](k)
$$

where the state $\left(p_{i}, q_{i}\right)$ identify the position of the front of the vehicle and the inputs $\left(v_{i}, w_{i}\right)$ account for the linear velocity
TABLE I

SENSITIVITY VALUES FOR EACH VEHICLE WITH UNKNOWN INITIAL POSITION.

\begin{tabular}{|c|c|c|c|c|c|}
\hline \# vehicle & 1 & 2 & 3 & 4 & 5 \\
\hline $\mathcal{S}\left(X_{i}(H), 1\right)$ & 2.0467 & 2.0654 & 2.1017 & 2.0428 & 2.0763 \\
\hline
\end{tabular}

and rotation. Moreover, $T$ stands for the sampling time, $\theta_{i}$ for the orientation and matrix $A_{i}\left(\theta_{i}\right)$ is given as:

$$
A_{i}\left(\theta_{i}\right)=\left[\begin{array}{cc}
\cos \theta_{i} & -l \sin \theta_{i} \\
\sin \theta_{i} & l \cos \theta_{i}
\end{array}\right] .
$$

In this scenario, the vehicles initial orientation is known to be $\theta(0)=\left[\begin{array}{lllll}\pi / 4 & \pi / 6 & \pi / 3 & \pi / 5 & -\pi / 3\end{array}\right]$ but their initial position is unknown with the constraint that $\forall_{1 \leq i \leq 5}$ : $\left\|\left[p_{i}(0) \quad q_{i}(0)\right]^{\top}\right\|_{\infty} \leq 1$. At each time step, after injecting their input, the nodes follow a consensus algorithm to decide the common position based on their state. Given the network topology and weights in Figure 1, one can define the iteration of a linear consensus algorithm of the type $x(k+1)=P x(k)$ to be given by the matrix:

$$
P=\operatorname{Adj} \otimes I
$$

and

$$
\operatorname{Adj}=\left[\begin{array}{ccccc}
0.20 & 0 & 0.45 & 0 & 0 \\
0.80 & 0.30 & 0 & 0 & 0 \\
0 & 0.35 & 0.10 & 0.50 & 0.60 \\
0 & 0.35 & 0 & 0.50 & 0 \\
0 & 0 & 0.45 & 0 & 0.40
\end{array}\right]
$$

In this example, the weights associated with each node contribution to the final value are $\left[\begin{array}{lllll}0.0826 & 0.0944 & 0.1468 & 0.0661 & 0.1101\end{array}\right] \otimes\left[\begin{array}{ll}1 & 1\end{array}\right]$. Therefore, the sensitivity analysis by traditional methods would have to take into consideration two different aspects. First, the inputs of each vehicle, even though equal, will have a different impact on their position depending on each initial orientation. On the other hand, the fact that those positions are subject to a consensus algorithm with different weights can change the relative impact of a node position to the final agreed one. Using the proposed strategy in this paper, the SVOs produce a set for the final output (as opposed to the example where the sensitivity was with respect to the state) and the inputs are the different signals to each of the vehicles.

The reported sensitivities are presented in Table I when the initial position of the nodes is unknown but their orientation is given by the initial state. These values translate that the largest amplitude in the final output is obtained when changing the vehicle 3 input.

In order to verify in an example the result in Lemma 1, we computed the sensitivity of the model to zero inputs and obtained $\mathcal{S}(X(H), 1)=2$, which was expected given that with no input the vehicles will not move and their final positions are going to be within the same initial state irrespective of how long it has passed since the initial time. The simulation was repeated with a known $\left(p_{i}(0), q_{i}(0)\right)$ taken from a uniform distribution by setting the initial polytope to 
TABLE II

SENSITIVITY VALUES FOR EACH VEHICLE WHEN THEIR INITIAL POSITION IS KNOWN AND UNIFORMLY DISTRIBUTED.

\begin{tabular}{|c|c|c|c|c|c|}
\hline \# vehicle & 1 & 2 & 3 & 4 & 5 \\
\hline $\mathcal{S}\left(X_{i}(H), 1\right)$ & 0.0467 & 0.0654 & 0.1017 & 0.0428 & 0.0763 \\
\hline
\end{tabular}

be a singleton. The new sensitivities are reported in Table II, which satisfies the result in Corollary 1 that states the sensitivity of considering all inputs to be the sum of taking one-factor-at-time. In order to confirm the result in Lemma 1, the general sensitivity for the unknown model was computed to be 2.3329 which is the sum of the sensitivities with known initial state and $\sum_{1 \leq \ell \leq 5} \mathcal{S}\left(X_{\ell}(H, \mathcal{D} \backslash\{\ell\}), 1\right)-4 \mathcal{S}_{x_{0}}$.

\section{CONCLUSIONS AND FURTHER RESEARCH}

This paper addressed the problem of computing the sensitivity of the state of a linear model to some inputs. By building on results from reachability analysis, a novel solution is proposed that computes the impact of inputs on the state by checking the maximum interval of realizations of the state found using two linear optimization programs. Simulations are presented for a case of a network of dynamical systems (vehicles modeled as unicycles) where different weights to the links measure various contributions to the overall output. Through simulation it is illustrated the main result of this paper that general sensitivity is equal to the sum of OAT approach only when the initial state is known. Two main directions of future work are envisioned: addressing the same issue on more complicated linear models (such as Linear Parameter-Varying) and resorting to other reachability tools; and, performing the same analysis for nonlinear and hybrid models using the general sensitivity based on their correspondent reachable sets.

\section{REFERENCES}

[1] A. Saltelli, K. Chan, E. M. Scott et al., Sensitivity analysis. Wiley New York, 2000, vol. 1.

[2] A. Saltelli, S. Tarantola, F. Campolongo, and M. Ratto, Sensitivity analysis in practice: a guide to assessing scientific models. John Wiley \& Sons, 2004.

[3] A. Saltelli, M. Ratto, T. Andres, F. Campolongo, J. Cariboni, D. Gatelli, M. Saisana, and S. Tarantola, Global sensitivity analysis: the primer. John Wiley \& Sons, 2008.

[4] D. M. Hamby, "A review of techniques for parameter sensitivity analysis of environmental models," Environmental Monitoring and Assessment, vol. 32, no. 2, pp. 135-154, Sep 1994.

[5] T. Turányi, "Sensitivity analysis of complex kinetic systems. tools and applications," Journal of Mathematical Chemistry, vol. 5, no. 3, pp. 203-248, Sep 1990.

[6] D. Silvestre, J. P. Hespanha, and C. Silvestre, "Fault detection for cyber-physical systems: Smart grid case," in 23rd International Symposium on Mathematical Theory of Networks and Systems (MTNS), Hong-Kong, China., July 2018.

[7] A. Saltelli, P. Annoni, I. Azzini, F. Campolongo, M. Ratto, and S. Tarantola, "Variance based sensitivity analysis of model output. design and estimator for the total sensitivity index," Computer Physics Communications, vol. 181, no. 2, pp. 259 - 270, 2010.

[8] T. A. Mara and S. Tarantola, "Variance-based sensitivity indices for models with dependent inputs," Reliability Engineering \& System Safety, vol. 107, pp. 115 - 121, 2012, sAMO 2010.

[9] D. G. Lainiotis and F. L. Sims, "Sensitivity analysis of discrete kalman filters," International Journal of Control, vol. 12, no. 4, pp. 657-669, 1970.
[10] R. Cukier, C. Fortuin, K. E. Shuler, A. Petschek, and J. Schaibly, "Study of the sensitivity of coupled reaction systems to uncertainties in rate coefficients. i theory," The Journal of chemical physics, vol. 59, no. 8, pp. 3873-3878, 1973.

[11] T. Pierce and R. Cukier, "Global nonlinear sensitivity analysis using walsh functions," Journal of Computational Physics, vol. 41, no. 2, pp. $427-443,1981$.

[12] A. Saltelli and I. M. Sobol', "About the use of rank transformation in sensitivity analysis of model output," Reliability Engineering \& System Safety, vol. 50, no. 3, pp. 225 - 239, 1995.

[13] A. Saltelli, S. Tarantola, and K. P.-S. Chan, "A quantitative modelindependent method for global sensitivity analysis of model output," Technometrics, vol. 41, no. 1, pp. 39-56, 1999.

[14] A. Saltelli, S. Tarantola, and F. Campolongo, "Sensitivity anaysis as an ingredient of modeling," Statist. Sci., vol. 15, no. 4, pp. 377-395, 112000.

[15] J. E. Oakley and A. O'Hagan, "Probabilistic sensitivity analysis of complex models: a bayesian approach," Journal of the Royal Statistical Society: Series B (Statistical Methodology), vol. 66, no. 3, pp. 751769, 2004.

[16] P. Doubilet, C. B. Begg, M. C. Weinstein, P. Braun, and B. J. McNeil, "Probabilistic sensitivity analysis using monte carlo simulation: A practical approach," Medical Decision Making, vol. 5, no. 2, pp. 157177, 1985, pMID: 3831638.

[17] A. van Griensven, T. Meixner, S. Grunwald, T. Bishop, M. Diluzio, and R. Srinivasan, "A global sensitivity analysis tool for the parameters of multi-variable catchment models," Journal of Hydrology, vol. 324, no. 1, pp. $10-23,2006$.

[18] X. Song, J. Zhang, C. Zhan, Y. Xuan, M. Ye, and C. Xu, "Global sensitivity analysis in hydrological modeling: Review of concepts, methods, theoretical framework, and applications," Journal of Hydrology, vol. 523 , pp. $739-757,2015$.

[19] N. Chitnis, J. M. Hyman, and J. M. Cushing, "Determining important parameters in the spread of malaria through the sensitivity analysis of a mathematical model," Bulletin of Mathematical Biology, vol. 70, no. 5, p. 1272, Feb 2008.

[20] K. Claxton, M. Sculpher, C. McCabe, A. Briggs, R. Akehurst, M. Buxton, J. Brazier, and T. O'Hagan, "Probabilistic sensitivity analysis for nice technology assessment: not an optional extra," Health Economics, vol. 14 , no. 4, pp. 339-347, 2005.

[21] I. A. Hiskens and M. A. Pai, "Trajectory sensitivity analysis of hybrid systems," IEEE Transactions on Circuits and Systems I: Fundamental Theory and Applications, vol. 47, no. 2, pp. 204-220, Feb 2000.

[22] E. Borgonovo and E. Plischke, "Sensitivity analysis: A review of recent advances," European Journal of Operational Research, vol. 248, no. 3, pp. $869-887,2016$.

[23] A. Saltelli, M. Ratto, S. Tarantola, and F. Campolongo, "Sensitivity analysis practices: Strategies for model-based inference," Reliability Engineering \& System Safety, vol. 91, no. 10, pp. 1109 - 1125, 2006, the Fourth International Conference on Sensitivity Analysis of Model Output (SAMO 2004).

[24] J. Shamma and K.-Y. Tu, "Set-valued observers and optimal disturbance rejection," IEEE Transactions on Automatic Control, vol. 44, no. 2, pp. $253-264$, feb 1999.

[25] D. Silvestre, P. Rosa, J. P. Hespanha, and C. Silvestre, "Stochastic and deterministic fault detection for randomized gossip algorithms," Automatica, vol. 78, pp. 46 - 60, 2017.

[26] — - "Fault detection for LPV systems using set-valued observers: A coprime factorization approach," Systems \& Control Letters, vol. 106, pp. $32-39,2017$.

[27] — "Set-based fault detection and isolation for detectable linear parameter-varying systems," International Journal of Robust and Nonlinear Control, vol. 27, no. 18, pp. 4381-4397, 2017, rnc.3814.

[28] — - "Self-triggered and event-triggered set-valued observers," Information Sciences, vol. 426, pp. $61-86,2018$.

[29] S. Keerthi and E. Gilbert, "Computation of minimum-time feedback control laws for discrete-time systems with state-control constraints," IEEE Transactions on Automatic Control, vol. 32, no. 5, pp. 432 435, may 1987.

[30] D. E. Hernández-Mendoza, G. R. Penaloza-Mendoza, and E. ArandaBricaire, "Discrete-time formation and marching control of multi-agent robots systems," in 2011 8th International Conference on Electrical Engineering, Computing Science and Automatic Control, Oct 2011, pp. 1-6. 